\title{
Ethnologies
}

\section{À propos d'une certaine muséologie en région}

\section{Philippe Dubé}

Volume 24, numéro 2, 2002

\section{Musées}

Museums

URI : https://id.erudit.org/iderudit/006644ar

DOI : https://doi.org/10.7202/006644ar

Aller au sommaire du numéro

\section{Éditeur(s)}

Association Canadienne d'Ethnologie et de Folklore

ISSN

1481-5974 (imprimé)

1708-0401 (numérique)

Découvrir la revue

Citer cet article

Dubé, P. (2002). À propos d'une certaine muséologie en région. Ethnologies, 24(2), 161-174. https://doi.org/10.7202/006644ar

\section{Résumé de l'article}

Sous forme de bilan des interventions menées en région dans le cadre des activités du Groupe de Recherche-Action en Muséologie à l'Université (GRAMUL), nous avons tenté par cet article de tirer sommairement les enseignements de terrain que cet exercice de praxis a autorisés. Ayant eu le mandat d'accompagner deux réseaux de musées au Québec (successivement en Sagamie et au Kamouraska) en vue de réfléchir avec les acteurs culturels du milieu muséal et patrimonial, nous avons, avec leur complicité, esquissé des perspectives d'avenir pour un horizon meilleur de fonctionnement. Cette recherche-action a d'abord permis de baliser le parcours de mise en réseau des musées en région en identifiant trois étapes de consolidation : l'esprit du lieu, les traces-témoins et la légende du pays. Une fois réalisée, il a fallu proposer un calibrage des forces en présence par une projection de spatialisation des spécialisations, question de faire écho plus fidèlement à la dynamique interne des réseaux constitués. Il reste maintenant à approfondir les divers aspects d'une muséologie tout-terrain que l'on pourrait énoncer pour les besoins du milieu dans une somme de principes applicables en micro-muséologie.
Ce document est protégé par la loi sur le droit d'auteur. L'utilisation des services d’Érudit (y compris la reproduction) est assujettie à sa politique d'utilisation que vous pouvez consulter en ligne.

https://apropos.erudit.org/fr/usagers/politique-dutilisation/ 


\title{
À PROPOS D'UNE CERTAINE MUSÉOLOGIE EN RÉGION
}

\author{
Philippe Dubé \\ Chercheur principal au GRAMUL \\ Professeur de muséologie, Université Laval
}

L'héritage ne se transmet pas, il se conquiert

(André Malraux, Commune, juillet 1935).

Au cours des dernières années, nous avons eu l'occasion de nous pencher sur diverses réalités muséales que l'on retrouve tout spécialement en région au Québec. À ce titre, deux mandats consécutifs ont été confiés au GRAMUL (Groupe de Recherche-Action en Muséologie à l'Université Laval) dans le but d'explorer - avec la complicité et la participation directe des acteurs culturels du milieu les possibilités de mise en réseau des institutions concernées. Deux territoires ont donc fait l'objet d'études exploratoires, le Saguenay-LacSaint-Jean (pour certains nommé la Sagamie) et le Kamouraska, et ce de 1997 à 2002.

Ces deux cas vont servir ici de toile de fond à une réflexion sur la richesse et la diversité des musées en région. Et nous allons voir, sous certains aspects, comment cette situation peut être comprise comme étant son parfait contraire, c'est-à-dire qu'elle peut être perçue - et surtout vécue - comme de la pauvreté et de la dispersion. En effet, au-delà du constat que nous avons fait en différents temps et lieux, il y a nécessairement des traits que nous avons dégagés d'un pareil examen.

L'éloignement par rapport aux grands équipements culturels, une démographie faible et décroissante, un taux de scolarisation généralement plus bas que celui des centres urbains et la vocation touristique du lieu qui se concentre en une seule saison ont obligatoirement des incidences sur la situation des musées en région. Et ce sont autant de facteurs que nous devons prendre en compte pour 
Groupe de Recherche-Action en Muséologie à l'Université Laval www.hst.ulaval.ca/gramul

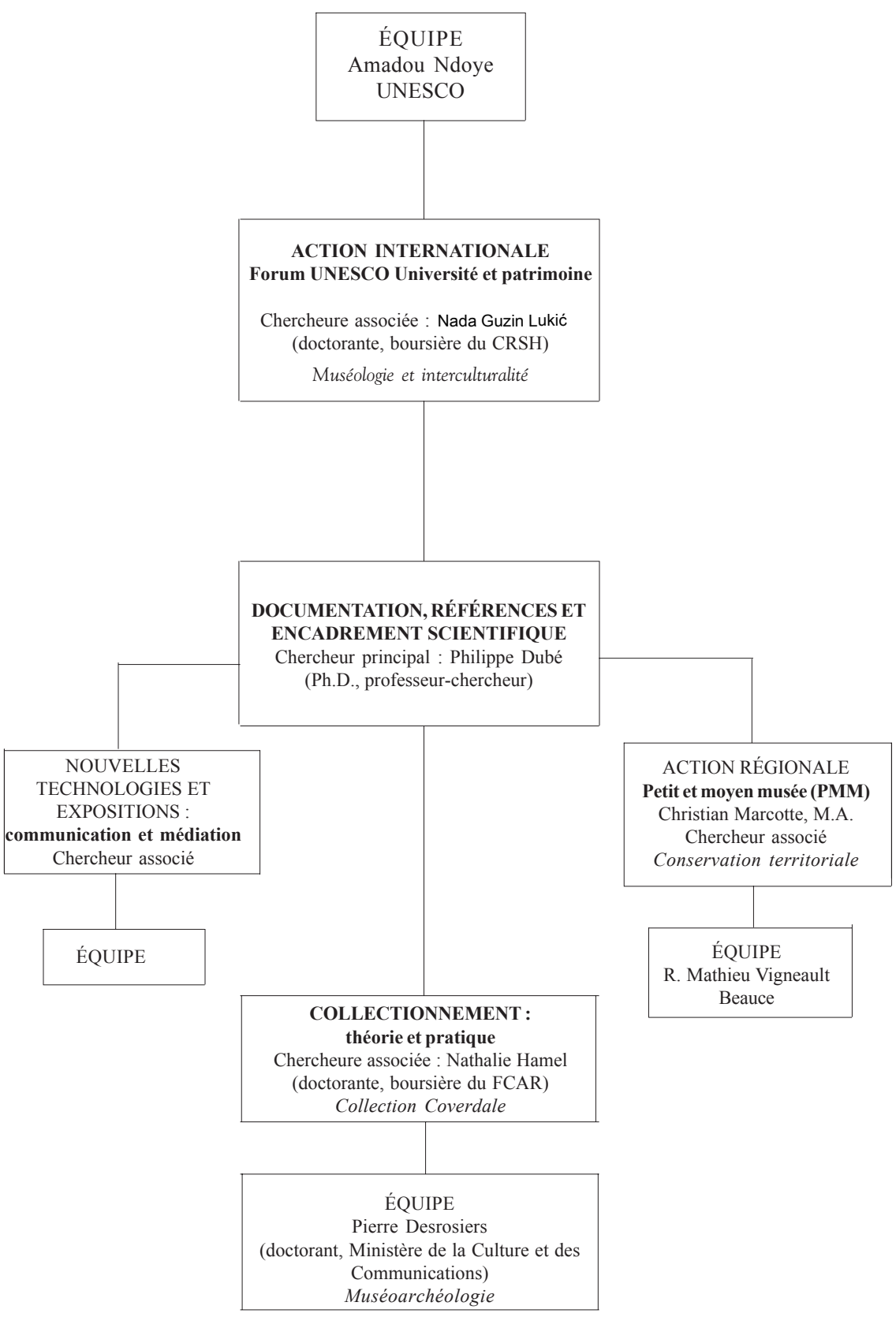


comprendre cette réalité à la fois simple et complexe (Collectif 1998). Nous allons ici tenter de saisir l'ampleur du phénomène, celle en somme d'une crise qui, selon un vieil adage, est foncièrement porteuse de solutions. Il s'agit là d'un enjeu majeur pour la muséologie québécoise actuelle ; à nous d'en cerner les contours, puis d'en saisir l'opportunité, celle de changer de paradigme de développement.

Il ne s'agit pas ici de monter un plaidoyer en faveur de la mise en réseau à tout prix des institutions muséales et patrimoniales en région, mais bel et bien de rendre compte, de façon critique, d'un travail accompli dans la perspective d'une nouvelle exploration de l'ingénierie culturelle en région.

\section{Une équipe en recherche-action}

Il faut d'abord situer ce travail dans une lignée d'actions qui gravitent autour d'une question axiale au sein du GRAMUL, à savoir : est-ce que le musée - tel qu'il est dans ses structures et son fonctionnement - a encore la capacité d'assumer honorablement la responsabilité patrimoniale dans une région culturelle donnée? Autrement dit, peutil, seul et articulé comme il l'est présentement, prendre à sa charge la conservation et la mise en valeur des patrimoines territoriaux? Voilà une problématique qui vise la compétence même de l'institution muséale en région (Weil 1990). Question que nous avons constamment remise sur notre table de travail, bien qu'elle se soit posée différemment à chaque nouveau cas depuis la fondation du GRAMUL en 1990 (Montpetit 2002).

Faut-il préciser que notre groupe est animé par quelques principes liés à la formation aux études de $2^{\mathrm{e}}$ et $3^{\mathrm{e}}$ cycles et à l'appui aux milieux culturels en région, principes qui, sans cesse, ont orienté la réalisation d'une vingtaine d'études au fil des années passées. Nous nous sommes penchés notamment sur des questions reliées au Petit et Moyen Musée (PMM) (Dubé 2001) qui nous ont permis de repenser intégralement son fonctionnement en créant un modèle que nous avons nommé les 3 X COM (Dubé 2001). À cette occasion, nous avons de plus élaboré une approche qui pourrait lui être éventuellement spécifique et que nous avons qualifiée de topomuséologique. Le chantier de préfiguration du Musée du Fjord notamment, mené de 1998 à 2000, a autorisé cette exploration vers de nouveaux modèles de fonctionnement que nous souhaiterions voir un jour en application, voire même en opération. 
Ces laboratoires, sous forme de chantiers, ont favorisé la formation d'équipes ponctuelles par projet et nous voudrions ici signaler la participation enthousiaste d'étudiant(e)s qui se sont prêté(e)s de bon gré au jeu de l'expérience scientifique. Nous voudrions aussi faire état de la réflexion menée par des professionnels des ministères (Gagnon 1993) qui nous ont fait confiance dans ces actions régionales.

\section{Un pays sans bon sens}

Pour les besoins de cette démonstration, nous avons dressé une compilation des enseignements tirés de notre expérience-terrain où l'enjeu de la mise en réseau des institutions muséales et patrimoniales est demeuré central dans notre questionnement. À priori, la région est au cœur de notre problématique puisqu'agissant comme une infrastructure sur laquelle repose et s'articule la dynamique culturelle d'un espace donné (Harvey 1994). Il faut donc tenter d'abord de la comprendre in abstracto et de voir comment elle peut, en tant qu'infrastructure, influer sur les acteurs locaux du patrimoine. Nous nous référons nommément à deux régions culturelles, soit le SaguenayLac-Saint-Jean et le Bas-Saint-Laurent, toutes deux chargées d'une histoire distinctement riche mais partageant globalement une situation commune de gestion patrimoniale, soit un déclin généralisé ; une décroissance progressive diront plus poliment d'autres.

Cette situation, quelle est-elle au juste? D'abord au chapitre de la démographie locale, on note une baisse graduelle enregistrée depuis plusieurs années.

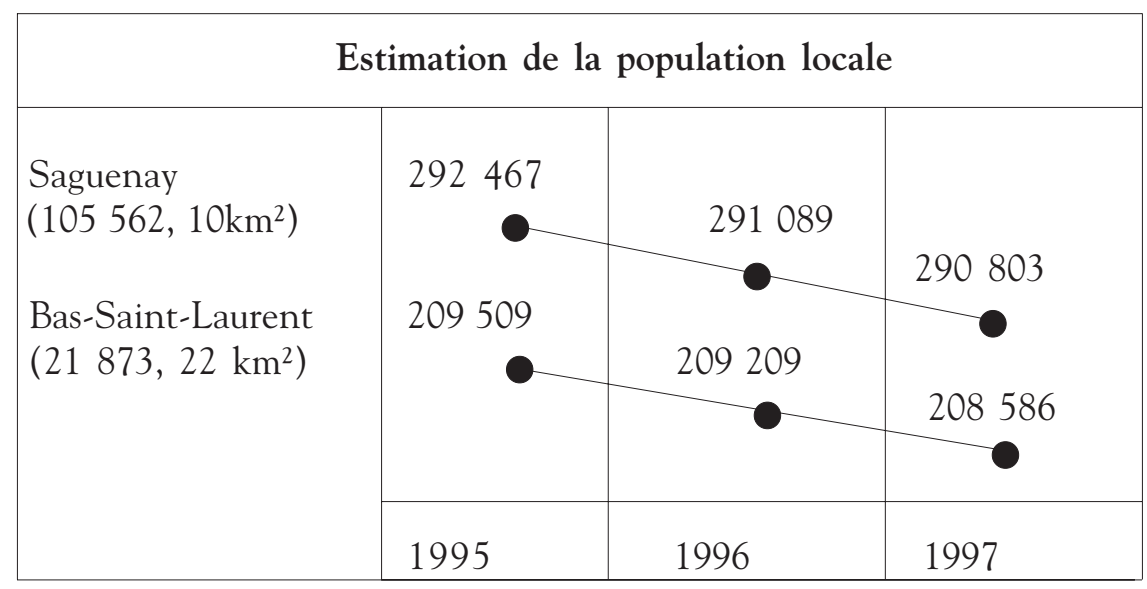




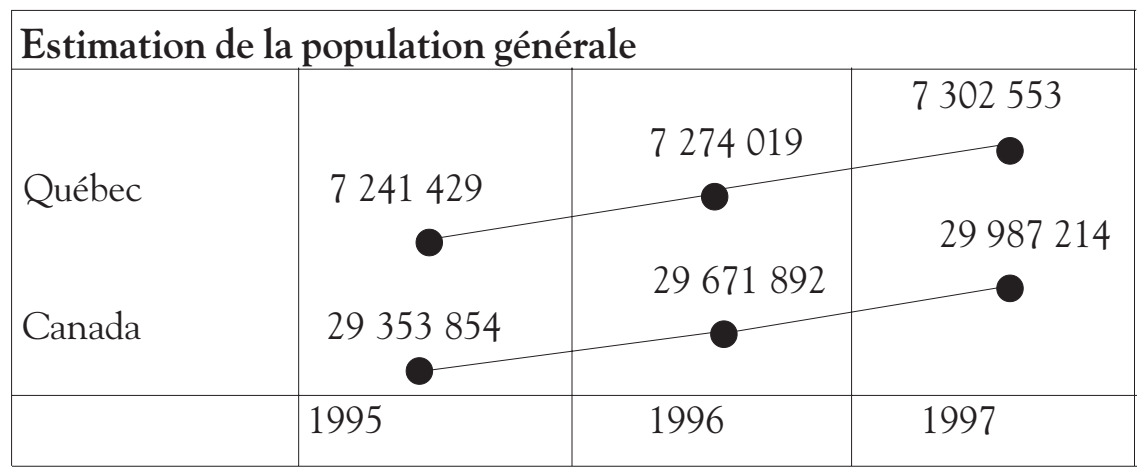

On assiste donc à un exode, à une réelle vidange des forces vives de ces régions. Cette déperdition, en termes de croissance, s'exprime également par un âge moyen qui peut accentuer les problèmes liés à la décroissance. À ce chapitre, on note que le Saguenay-Lac-Saint-Jean jouit encore d'une certaine jeunesse mais, en comparaison avec d'autres paramètres à prendre en compte, il s'agit là d'une bien mince consolation.

\begin{tabular}{|l|c|}
\hline Régions & Âge moyen \\
\hline Bas Saint-Laurent & 37,4 ans \\
Québec & 36,3 ans \\
Canada & 35,8 ans \\
Saguenay & 35,1 ans \\
\hline
\end{tabular}

Globalement, la population vieillit, atteignant parfois des moyennes inquiétantes. Par ailleurs, l'âge avancé peut être un indice favorable pour la fréquentation des musées et lieux du patrimoine, mais à la condition que la scolarité soit tout de même assez élevée.

\begin{tabular}{|l|c|c|}
\hline Scolarité & $\begin{array}{c}\text { Diplôme d'études } \\
\text { universitaires }\end{array}$ & $\begin{array}{c}\text { Pas d'études } \\
\text { secondaires }\end{array}$ \\
\hline Canada & $17,4 \%$ & $13,4 \%$ \\
Québec & $16,9 \%$ & $20,4 \%$ \\
Saguenay & $12,3 \%$ & $19,9 \%$ \\
Bas-Saint-Laurent & $11,4 \%$ & $26,8 \%$ \\
\hline
\end{tabular}


Quand on y regarde de près, peu de personnes ont terminé des études universitaires en région, si on compare cette moyenne au niveau national, tout comme, à l'autre extrême, la part de la population n'ayant pas fait d'études secondaires, ce qui n'est généralement pas un gage de support aux activités culturelles. Tout ceci mérite évidemment d'être nuancé, pondéré, mais pour les besoins de cette démonstration, par ces indicateurs on vient souligner que le déclin progressif de deux régions reste à prendre en compte globalement.

On ne reprendra pas ici l'argumentaire qui fait la preuve, de plus en plus éclatante, du sous-financement culturel en région puisqu'il a maintes fois été démontré depuis la parution de l'article de Jeanne Morazain dans Le Devoir du 11 mai 1991, sous le titre "Le sous-financement chronique des régions » et suivi du dépôt, en novembre 1991, du Mémoire présenté à la Commission parlementaire sur la culture par Jean-Paul Thomin et Suzanne Chassé intitulé Le soutien des institutions régionales au Québec: un levier essentiel pour la démocratisation de la culture. Et, comme le signalait si justement Gaston Gagnon de la Direction régionale du Saguenay-Lac-Saint-Jean au ministère de la Culture et des Communications du Québec, dans une étude réalisée il y a maintenant plus de dix ans :

Le Québec à deux vitesses, l'un qui s'enrichit et l'autre qui s'appauvrit, décrit dans le mémoire de 1989 du Conseil des affaires sociales est donc bien réel sur le plan culturel. Mais pour contrer cette dualité et rejoindre un des objectifs du Groupe-Conseil sur la Politique culturelle du Québec [depuis lors adoptée], qui est de favoriser l'accès des Québécois à la vie culturelle sur l'ensemble du territoire, la mise en commun des ressources devient, avec le droit à la parité des équipements, un impératif de premier plan à l'innovation créatrice et au déploiement d'une stratégie de communication (Gagnon 1991).

En effet, si l'exotisme d'une destination - créé souvent par la distance qui la sépare des grands centres urbains - joue en faveur d'un développement touristique, l'éloignement engendre un coût réel sur le plan culturel. D'ailleurs, on évalue mal les aspects positifs de la proximité des équipements culturels qui incluent les bibliothèques d'importance, les centres documentaires, les universités et leurs équipes de recherche, les grandes collections, les éditeurs, etc... autant d'acteurs qui créent de la culture, surtout à caractère patrimonial. L'agglomération des ressources a certes un effet de nucléarisation sur toute dynamique culturelle. Sans mentionner que la dispersion - pour ne pas évoquer 
la disparité qui souvent l'accompagne - des équipements, quand il s'agit de couvrir un vaste espace où la population est le plus souvent parsemée, rend difficile l'effervescence pourtant nécessaire à la culture. Cet élément de géospatialité vient porter un dur coup (coût) à l'action patrimoniale sur un territoire donné. Il faut croire que le voisinage assure une concentration qui favorise le développement, sans parler ici d'émulation qui stimule une saine concurrence dans tous les domaines. C'est ce qu'on appelle l'économie d'agglomération que l'on ne peut pas ignorer dans le contexte étudié.

Autant de facteurs qui doivent être pris en compte quand on établit les paramètres d'une évaluation qui se veut objective. Ceci dit, à ressources moindres, nécessité fait loi. Et c'est là où intervient la mobilisation du milieu, alliée au nécessaire support étatique qui, enfin, permet d'explorer des avenues possibles de solution envers un contredéveloppement, une situation de réelle contre-production culturelle.

\section{La réthorique des artefacts}

C'est au moment du « déluge » dans le Saguenay à l'été de 1996, surnommé pour l'occasion «Juillet noir » que, sur l'invitation du ministère de la Culture, nous avons été appelé à réfléchir sur la situation des musées en région, situation qu'avait aggravée la catastrophe écologique. Au terme d'une année de travail avec les acteurs du lieu se dégageait un élément capital de compréhension globale de la situation des musées de la région de la Sagamie (Dubé 1997). Au-delà de la grande diversité des institutions étudiées qui semblait former un tout assez cohérent, sinon complémentaire, et pouvait à la limite s'exprimer comme un ensemble sous la forme théorique d'un Musée-Territoire, nous devions trouver le dénominateur commun aux seize institutions le composant. Et cet élément a été d'identifier un thème général, englobant mais propre à tous les musées, et qui allait constituer la spécificité du lieu. Une thématique unificatrice, un thème donc intégrateur, permettant de faire une lecture de synthèse du territoire culturel en question. En Sagamie, par exemple, nous avons convenu collectivement que la concentration des forces hydrauliques du lieu était déterminante pour «expliquer» le lieu. Tout le développement régional pouvait en effet se comprendre par la forte présence du bassin de ses eaux : le lac et la rivière. Et, qui plus est, cette région était fondée sur une rupture par rapport à la société traditionnelle, ce qui nous a 
Mise en espace / Mise en contexte / Mise en récit

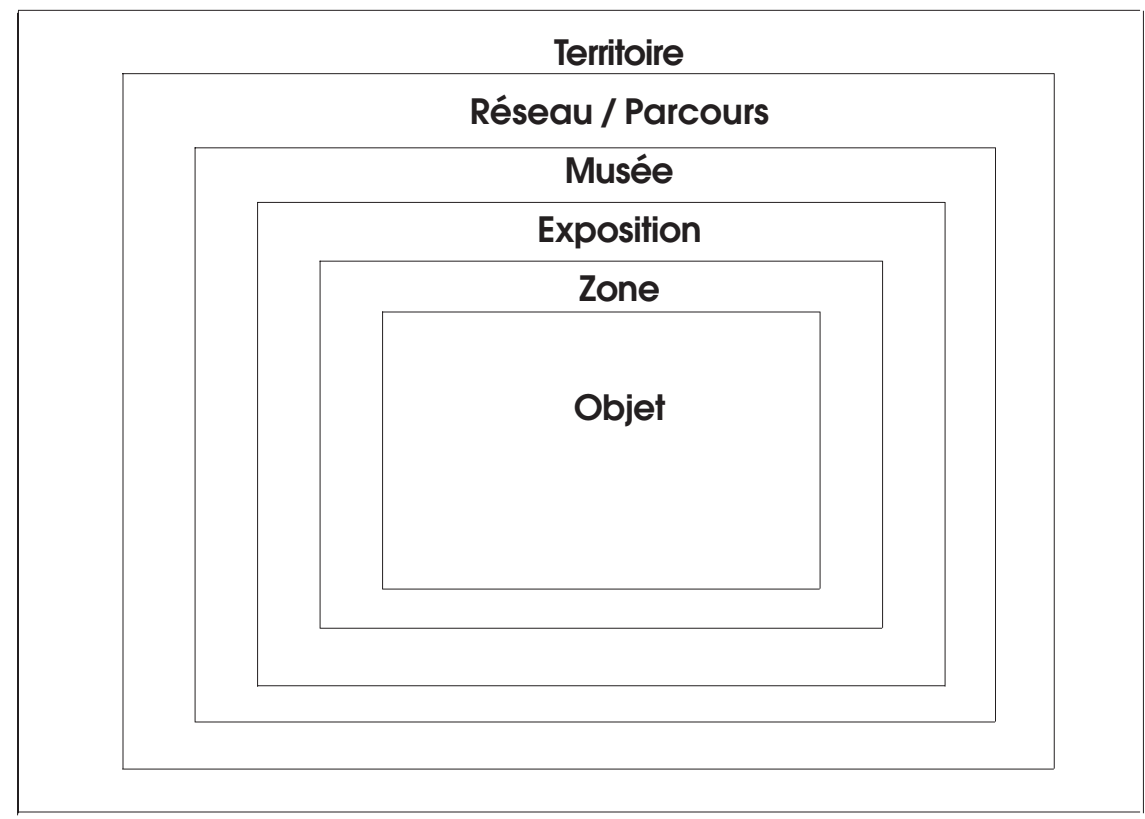

\section{Pour une certaine méthodologie :}

Étapes préalables à la mise en réseau des institutions muséales et patrimoniales dans la perspective de création d'un Musée-Territoire

L'esprit du lieu

1. Dégager un «thème

de synthèse * par l'examen exhaustif des institutions muséales et patrimoniales d'un territoire culturel donné (mission, profil, historique, collections, etc...)
Les traces témoins

2. Procéder à une des c o 11 e c t i o n s régionales en vue d'identifier le «trésor territorial» par un examen critique des éléments du patrimoine, tant intra muros qu'extra muros
La légende du pays

3. Élaborer, sur la base de résultats obtenus (1 et 2) un « récit patrimonial " auquel viendrait contribuer chacune des composantes du réseau en formation 
amené à la qualifier de "laboratoire de la modernité » dans le Québec des $\mathrm{XIX}^{\mathrm{e}}$ et $\mathrm{XX}^{\mathrm{e}}$ siècles.

Ce premier constat nous a conduit vers une deuxième étape de notre recherche qui nous a plongé cette fois dans les profondeurs, les entrailles culturelles du territoire, alors que nous allions tenter d'identifier le trésor régional en sélectionnant les objets révélateurs du lieu, venant ainsi renforcer notre première lecture de synthèse. Et c'est sur la base de ce vaste corpus d'artefacts (que nous avions limités au nombre de 150 objets signifiants, une dizaine par institution environ) que nous pouvions tenter de dépasser la seule lecture de synthèse pour enfin passer à l'élaboration d'un récit. Récit qui, non seulement donne de la cohérence à la compréhension du lieu, mais offre aussi un élément de liaison entre les différents partenaires à mettre en réseau (Vigneault 2000).

\section{Le retour de la légende}

Fort de cette expérience liée à l'exercice de mise en réseau et, de manière ultime, à la création d'un réel Musée-Territoire, nous avons pu mettre à l'épreuve, au cours de l'an 2000-2001, la méthode que nous avions articulée au départ en Sagamie, mais cette fois transposée dans un autre contexte, celui du Kamouraska. Il fallait non seulement revoir les composantes du réseau potentiel à travers un examen exhaustif des ressources, mais aussi penser le lieu en termes de développement culturel et ce, sur une base pratique et réalisable à moyens termes (Marcotte et Vigneault 2002). D'où l'importance accordée à la mise en place d'une structure qui pouvait éventuellement porter le projet d'une mise en réseau intégré en tablant sur la force du récit, la puissance de la légende, au sens le plus fort du terme. D'abord parce qu'il s'agit avant tout d'une forme de récit populaire plus ou moins fabuleux qui demeure le véhicule idéal pour transporter la rumeur. Et, deuxièmement, parce que la légende est ce texte qui accompagne toute image, toute illustration et lui donne enfin son sens. Le mode narratif est en soi porteur parce qu'il offre cohérence (du récit) et cohésion (des partenaires) afin d'agir de manière concertée dans le champ patrimonial.

Mais une des garanties de son succès était de le penser en fonction du rééquilibrage des forces en présence, du re-calibrage du rôle de chacune des institutions dans cette restructuration. C'est donc à une reconfiguration des acteurs régionaux que nous les avons invités, où 


\section{MUSÉE-TERRITOIRE :}

Nouveau paradigme de développement patrimonial en région

Spatialisation des spécialisations muséales sur le territoire

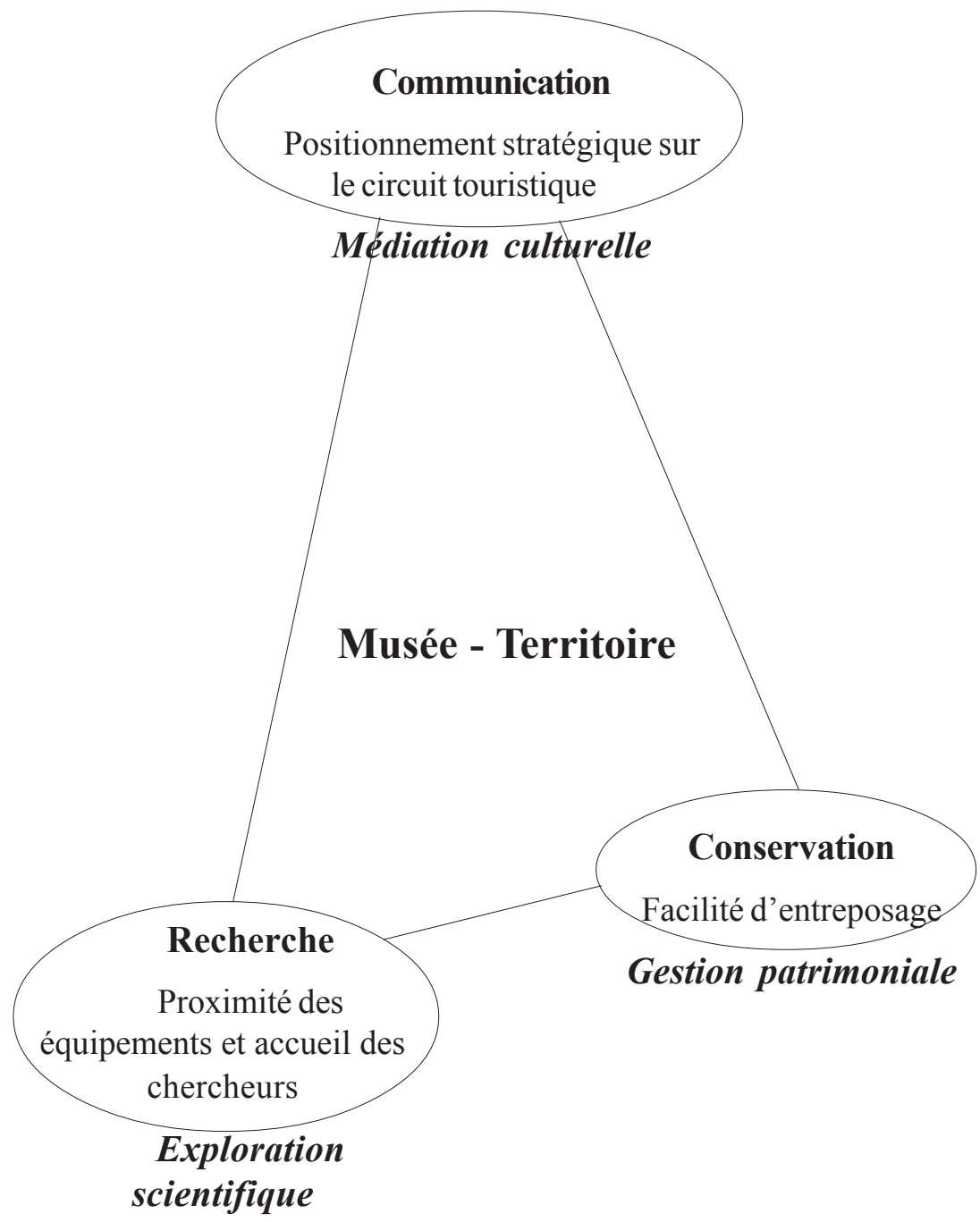


certains devront se concentrer sur un aspect plus spécifique de la muséologie pour enfin assurer un fonctionnement viable au MuséeTerritoire. C'est ainsi que nous proposons une spatialisation des spécialisations muséales sur le territoire où, par la cohérence des jeux de rôle, nous espérons atteindre la cohésion d'action. Voilà un nouveau modèle qui exige une révision radicale du rôle de chacun afin de créer une unité harmonique qui autorisera une action à géométrie variable, mais centrée sur un objectif commun et territorial. Recentrant sur une fonction plus spécifique, on vient de redresser la dispersion et, qui sait, vivre enfin la diversité comme une réelle richesse.

\section{Un état de crise}

On a vu tout au long de cette brève démonstration que les musées en région vivent dans leur pratique quotidienne de grandes difficultés de fonctionnement, sinon témoignent d'un certain malaise. Malaise à simplement remplir honorablement leur mandat patrimonial, celui d'assurer en fait la veille, une action de vigie sur l'héritage d'un lieu, celui d'une communauté vivante, celui en somme d'un territoire culturel donné.

D'emblée nous assumons, avec le milieu professionnel en place, que cet état de crise oblige à prendre un virage salutaire. Virage certes à prendre, mais à prendre dans quelle direction au juste ? Rien n'est plus simple et à la fois plus complexe que d'identifier cette nouvelle orientation. Mais le milieu est somme toute condamné à la trouver et c'est ce que nous avons tenté de faire au cours des cinq dernières années en l'accompagnant, avec sa généreuse complicité, vers les voies de la mise en réseau intégré et regroupé autour d'un récit, au cœur de la légende du pays.

Faut-il ici rappeler que le mot crise nous vient du grec krisis emprunté celui-là au vocabulaire médical décrivant « la phrase décisive d'une maladie » ? C'est donc que tout état de crise en appelle à une décision pour atteindre enfin son dénouement, sa conclusion, qu'elle soit négative ou positive. Et si l'on se réfère cette fois au japonais, le mot crise est composé de deux kanjis — deux idéogrammes - qui expriment très justement l'idée que la solution réside dans le creuset du problème. Le mot japonais est d'abord constitué de danger, que représente ce qui menace, ce qui met en péril l'objet même de la maladie. Le sujet malade est ici aux prises avec son mal. Mais pour créer le mot crise, on doit 


\section{La crise}
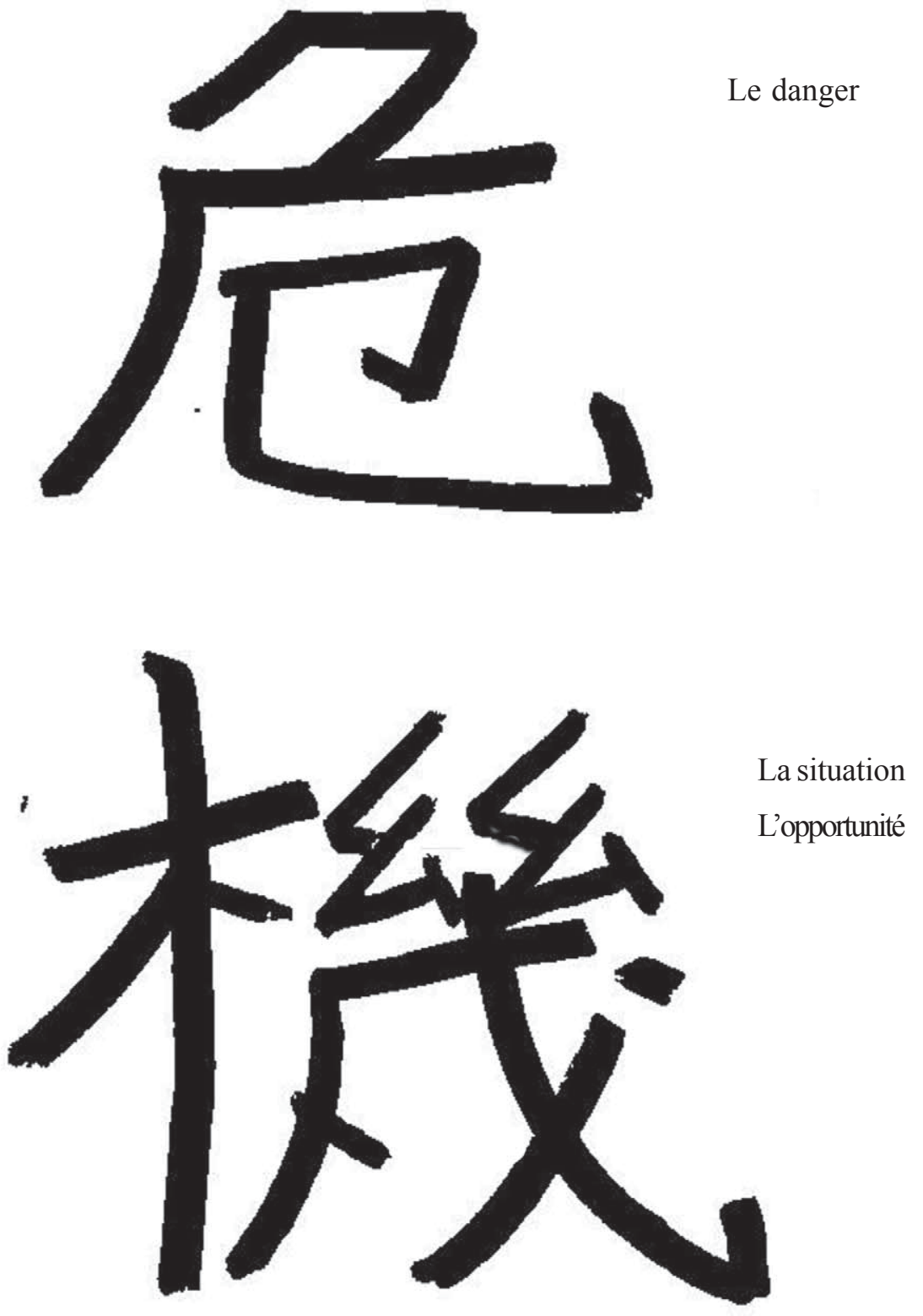
obligatoirement lui accoler l'idéogramme signifiant l'opportunité, donc la situation qui peut engendrer la solution. Le remède qui va donc procéder enfin à l'éradication du mal. À cet état de crise, au terme de cette "phase décisive ", se trouve donc une solution, une issue qui permet d'entrevoir une sortie à l'impasse. Et comme le dit laconiquement William James, ce bel esprit du Nouveau Monde, «c'est dans les chances qu'il peut saisir que vit le cœur humain ». Et cette chance est ici à notre portée, il s'agit maintenant d'en prendre conscience, de la saisir et enfin d'agir. 


\section{Références}

Collectif, 1998, Le village, tout un patrimoine, Actes du colloque des 8 et 9 février 1998 à Rimouski, Nicolet, Solidarité rurale du Québec. Dubé, Philippe, 2001, «Le patrimoine territorial : ethnologie et muséologie ", dans Pichette, Jean-Pierre et al., Entre Beauce et Acadie, Québec, Presses de l'Université Laval : 422-434.

_ 2001 , «Vers un nouveau modèle générique du Petit et Moyen Musée », Muse, vol. XIX (1) : 8-9.

— 1997, Étude exploratoire de mise en réseau des lieux muséaux et patrimoniaux du Saguenay Lac-Saint-Jean, Cahier 1, Québec, GRAMUL.

Gagnon, Gaston, 1993, Le patrimoine au Saguenay-Lac-Saint-Jean aux sources de l'identité régionale, Québec et Chicoutimi, Ministère de la culture.

—, décembre 1991, Le patrimoine du Saguenay-Lac-Saint-Jean : projet de mise en réseau, Séminaire de synthèse MSL-68000, Université du Québec à Montréal.

Grimwade, Gordon et Bill Carter, 2000, « Managing Small Heritage Sites with Interpretation and Community Involvement ", International Journal of Heritage Studies, vol. 6 (1) : 33-48.

Harvey, Fernand (dir.), 1994, La région culturelle, problématique interdisciplinaire, Québec, CEFAN / IQRC.

Lemieux, Denise (dir.), 2002, Traité de la culture, Québec, Éditions de l'IQRC et Presses de l'Université Laval.

Marcotte, Christian et R. Mathieu Vigneault, sous la direction de Philippe Dubé, 2002, La mise en réseau des institutions muséales et patrimoniales du Kamouraska, Québec, GRAMUL.

Montpetit, Raymond, 2002, "Musées et muséologie, un champ de recherche dynamique en émergence », dans Lemieux, Denise (dir.), Traité de la culture, Québec, Éditions de l'IQRC et Presses de l'Université Laval : 90.

Thomin, Jean-Paul et Suzanne Chassé, novembre 1991, Le soutien des institutions régionales au Québec: un levier essentiel pour la démocratisation de la culture, Mémoire présenté à la Commission parlementaire sur la culture du Québec.

Vigneault, R. Mathieu, 2000, Récit de la Sagamie : territoire et patrimoine, Québec, GRAMUL.

Weil, Stephen E., 1990, Rethinking the Museum and other Meditations, Washington, Smithsonian Institution Press. 